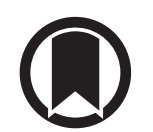

CrossMark

\title{
Extracorporeal membrane oxygenation for acute respiratory distress syndrome due to Pneumocystis pneumonia
}

\begin{abstract}
To the Editor:
Pneumocystis jirovecii pneumonia (PcP) occurs exclusively in immunocompromised patients. About $50 \%$ of $\mathrm{PcP}$ is HIV related, the other half is associated with immunosuppression for other reasons [1]. If PcP progresses to an acute respiratory distress syndrome (ARDS) requiring intensive care and invasive mechanical ventilation, the prognosis is generally poor [1] and mortality is about $80 \%$ if additional veno-venous extracorporeal membrane oxygenation (VV-ECMO) support is necessary [1]. Despite lack of clear evidence [2], VV-ECMO has become an integral part in the rescue therapy of severe ARDS. Moreover, some centres start VV-ECMO at early time-points in order to rigorously follow (ultra-) protective ventilation strategies [3]. So far, VV-ECMO in patients with PcP-associated ARDS has been reported only on the basis of singular case reports, including one case of awake ECMO [4]. Using ECMO in patients who are awake and spontaneously breathing might avoid complications associated with sedation and invasive mechanical ventilation. Our group was the first to describe this awake ECMO approach in a bridge-to-transplant setting $[5,6]$ as well as in a small number of ARDS patients $[7,8]$. However, evidence on the safety and efficacy of awake VV-ECMO strategies in this population is lacking [7]. PcP classically leads to an isolated single organ failure without accompanying systemic complications such as septic shock with haemodynamic instability or acute kidney injury (AKI). At the same time, these patients often require prolonged invasive mechanical ventilation with a high risk of complications including ventilator-associated pneumonia and pneumothorax. Hence, patients with PcP and severe hypoxaemia may be candidates for an awake ECMO strategy as a bridge to recovery.
\end{abstract}

We retrospectively analysed patients with PcP-associated ARDS who required VV-ECMO (alveolar oxygen tension $\left(\mathrm{PaO}_{2}\right) /$ inspiratory oxygen fraction $\left(\mathrm{FIO}_{2}\right)<100$ or $\left.\mathrm{pH}<7.15\right)$ in our tertiary care hospital from 2010 to 2018. Written informed consent was waived by the local ethics committee. 16 patients were identified, of whom six (38\%) were treated with initial (no intubation prior to ECMO initiation) awake VV-ECMO when deemed cooperative and expected to tolerate the awake concept.

The two cohorts (intubated VV-ECMO and awake VV-ECMO) were comparable in terms of most demographic and clinical parameters. Median age of patients was 41 (33-67) years and the majority was male (75\%). Primary cause for PcP infection was HIV in six (38\%) patients and medical immuno-suppression in the other 10 patients. All patients were receiving intravenous trimethoprim-sulfamethoxazole at high daily doses of 26 (17.9-33.5) mg.kg ${ }^{-1}$ body weight and additional daily prednisolone at $1 \mathrm{mg} \cdot \mathrm{kg}^{-1}$ body weight. With regard to organ failure, the majority of patients had predominantly singular pulmonary dysfunction indicated by rather low rates of vasopressor support $(31 \%)$ and renal replacement therapy $(25 \%)$ as well as physiological serum lactate concentrations $\left(1.6(1.3-2.2) \mathrm{mmol} \cdot \mathrm{L}^{-1}\right)$. Median oxygenation indices at initiation were 68 and $72(\mathrm{p}=0.76)$ with median $\mathrm{pH}$ values of $7.37(7.18-7.42)$ and $7.38(7.32-7.42)(\mathrm{p}=0.46)$ for intubated and awake ECMO patients, respectively. Of the six non-intubated patients, non-invasive ventilation and nasal high flow cannula oxygenation were employed for three patients each. The median Sequential Organ Failure Assessment (SOFA) scores were 11 and 7, respectively, with the difference between both groups attributable to the use of sedatives in ventilated patients. Renal replacement therapy requirement was not different, as were C-reactive protein and lactate dehydrogenase concentrations. The burden of

@ERSpublications

Extracorporeal membrane oxygenation (ECMO) may be justified in immunocompromised patients with Pneumocystis-associated acute respiratory distress syndrome, and an awake ECMO strategy might be feasible in selected patients http://bit.ly/2YFyrOK

Cite this article as: Stahl K, Schenk H, Seeliger B, et al. Extracorporeal membrane oxygenation for acute respiratory distress syndrome due to Pneumocystis pneumonia. Eur Respir J 2019; 54: 1900410 [https://doi. org/10.1183/13993003.00410-2019]. 
comorbidities was similar in both cohorts. However, sedated patients needed vasopressors in $50 \%$ of cases at moderate doses whereas primarily awake ECMO patients were all haemodynamically stable $(\mathrm{p}=0.04)$.

Median respiratory frequency $(f \mathrm{R})$ in the awake ECMO group was 37 (31-40) breaths $\mathrm{min}^{-1}$ before ECMO implantation and 22 (19-26), 26 (18-33), and $22(20-24)$ breaths $\cdot \mathrm{min}^{-1}$ at $4 \mathrm{~h}, 24 \mathrm{~h}$ and $48 \mathrm{~h}$ thereafter, respectively.

The overall survival until discharge from hospital was five (31\%) out of 16 . Four (67\%) of the six patients who were primarily treated with the awake ECMO support survived until discharge from hospital. Although, out of these six primarily awake ECMO patients, four required secondary intubation after 5 (1.5-14) days, the survival of this awake cohort was strikingly better compared to primarily intubated ECMO patients (10\%; $\mathrm{p}=0.011$ (figure 1). Of note, both awake ECMO patients who died required secondary intubation during the course of their intensive care unit stay. Causes of death in the awake ECMO group were pulmonary bleeding $(n=1)$ and irreversible lung failure $(n=1)$. In the intubated ECMO group, progressive secondary septic shock due to superinfections $(n=5)$ as well as lack of ARDS improvement $(n=3)$ and fatal pulmonary bleeding $(n=1)$ were noted. On univariate regression analysis, awake VV-ECMO strategy was a strong predictor for survival in our cohort of PcP patients (OR 18, 95\% CI 1.2-260.9; $\mathrm{p}=0.034)$.

To the best of our knowledge, this is the largest cohort of PcP patients treated with ECMO reported so far. Although mortality remained high, our data suggest that the use of ECMO is not necessarily futile in this immunocompromised patient population. In addition, a primarily awake ECMO strategy seems feasible in selected patients. However, criteria to identify ideal candidates have not been established and depend on the expertise of a given centre. It is not uncommon that patients who are primarily awake require later secondary intubation, mostly for agitation and delirium, or worsening of oxygenation despite maximal ECMO support.

We acknowledge the limitations of this study, in particular the small number of patients, the single-centre setting and the retrospective design. The sample size was too small for a matched pairs analysis or a propensity adjusted analysis to control for a possible selection bias. The observation that patients in the awake ECMO group were haemodynamically stable at baseline might represent such a bias. Thus, drawing generalisable conclusions from the comparison of outcomes between the two groups is difficult.

The comparison of ARDS severity of intubated and non-intubated patients by means of oxygenation index was confounded by the proportion of patients on high-flow nasula cannula.

One advantage of awake non-intubated ECMO might theoretically be the avoidance of ventilator-induced lung injury but, at the same time, vigorous spontaneous breathing efforts have also been demonstrated as potentially injurious: termed "self-inflicted lung injury" [1,9]. In this retrospective analysis, we cannot

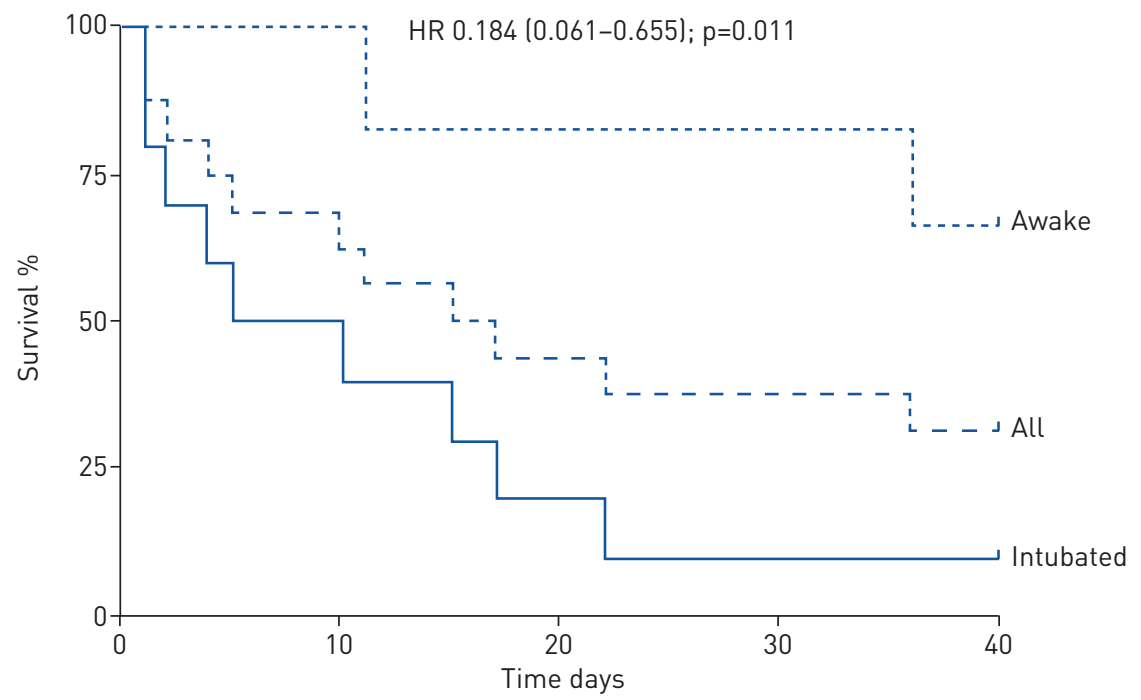

FIGURE 1 Survival in intubated versus awake veno-venous extracorporeal membrane oxygenation (VV-ECMO) patients with Pneumocystis pneumonia (PcP). Kaplan-Meier graphs showing the 40-day survival course in awake $(n=6)$, intubated $(n=10)$ and all VV-ECMO patients with PCP-associated acute respiratory distress syndrome (mortality awake ECMO two (33\%) out of six versus intubated ECMO nine $(90 \%)$ out of $10 ; \mathrm{p}=0.01$ ). 
address this important concern but we did observe that respiratory rates following ECMO implantation declined by over $40 \%$ indicating a clinical relief of patients' respiratory distress.

Despite these limitations, our findings support the notion that ECMO support may be justified in immunocompromised patients with PcP-associated ARDS and suggest that an awake ECMO strategy might be feasible in selected patients. Whether the avoidance of intubation and mechanical ventilation is associated with better outcome, is however unclear. We hope that these data might stimulate future prospective studies on the use of ECMO in patients with $\mathrm{PcP}$-associated lung failure.

Klaus Stahl $\oplus^{1,6}$, Heiko Schenk $\oplus^{2,6}$, Benjamin Seeliger ${ }^{3}$, Olaf Wiesner ${ }^{3}$, Julius J. Schmidt ${ }^{2}$, Johann Bauersachs ${ }^{4}$, Tobias Welte ${ }^{3}$, Christian Kühn ${ }^{5}$, Axel Haverich ${ }^{5}$, Marius M. Hoeper $\circledast^{3}$ and Sascha David ${ }^{2}$

${ }^{1}$ Dept of Gastroenterology, Hepatology and Endocrinology, Hannover Medical School, Hannover, Germany. ${ }^{2}$ Division of Nephrology and Hypertension, Hannover Medical School, Hannover, Germany. ${ }^{3}$ Dept of Respiratory Medicine and German Centre of Lung Research (DZL), Hannover Medical School, Hannover, Germany. ${ }^{4}$ Dept of Cardiology and Angiology, Hannover Medical School, Hannover, Germany. ${ }^{5}$ Dept of Cardiothoracic, Transplant and Vascular Surgery, Hannover Medical School, Hannover, Germany. ${ }^{6}$ Authors contributed equally to the manuscript and are both considered first authors.

Correspondence: Sascha David, Dept of Nephrology, Hannover Medical School, Carl-Neuberg-Str. 1, Hannover, Niedersachsen, 30655, Germany. E-mail: david.sascha@mh-hannover.de

Received: 01 March 2019 | Accepted after revision: 12 May 2019

Author contributions: K. Stahl, H. Schenk, B. Seeliger, J.J. Schmidt and S. David obtained retrospective data. K. Stahl, H. Schenk, J. Bauersachs, T. Welte, C. Kühn, M.M. Hoeper, A. Haverich and S. David analysed and discussed the data and generated the figure. S. David and K. Stahl wrote the manuscript; all authors proof-read the manuscript.

Conflict of interest: K. Stahl has nothing to disclose. H. Schenk has nothing to disclose. B. Seeliger has nothing to disclose. O. Wiesner has nothing to disclose. J.J. Schmidt has nothing to disclose. J. Bauersachs has nothing to disclose. T. Welte reports personal fees or lectures/advisory board work from AstraZeneca, Boehringer, Berlin Chemie, Chiesi, GSK and Novartis, grants from AstraZeneca and Novartis, outside the submitted work. C. Kühn has nothing to disclose.

A. Haverich has nothing to disclose. M.M. Hoeper reports personal fees for lectures and consultancy from Actelion, Bayer, MSD and Pfizer, outside the submitted work. S. David has nothing to disclose.

Support statement: S. David is supported by the German Research Foundation (DA 1209/4-3) and by the German Lung Centre (DZL). J. Bauersachs, C. Kühn, M.M. Hoeper and A. Haverich were supported by the German Research Foundation (DFG), Clinical Trails Research Group (KFO 311) "(Pre)terminal heart and lung failure: Unloading and repair".

\section{References}

1 Schmidt JJ, Lueck C, Ziesing S, et al. Clinical course, treatment and outcome of Pneumocystis pneumonia in immunocompromised adults: a retrospective analysis over 17 years. Crit Care 2018; 22: 307.

2 Combes A, Hajage D, Capellier G, et al. Extracorporeal membrane oxygenation for severe acute respiratory distress syndrome. N Engl J Med 2018; 378: 1965-1975.

3 Davis JL, Morris A, Kallet RH, et al. Low tidal volume ventilation is associated with reduced mortality in HIV-infected patients with acute lung injury. Thorax 2008; 63: 988-993.

4 Lee N, Lawrence D, Patel B, et al. HIV-related Pneumocystis jirovecii pneumonia managed with caspofungin and veno-venous extracorporeal membrane oxygenation rescue therapy. BMJ Case Rep 2017; 2017: bcr2017221214.

5 Fuehner T, Kuehn C, Hadem J, et al. Extracorporeal membrane oxygenation in awake patients as bridge to lung transplantation. Am J Respir Crit Care Med 2012; 185: 763-768.

6 Olsson KM, Simon A, Strueber M, et al. Extracorporeal membrane oxygenation in nonintubated patients as bridge to lung transplantation. Am J Transplant 2010; 10: 2173-2178.

7 Hoeper MM, Wiesner O, Hadem J, et al. Extracorporeal membrane oxygenation instead of invasive mechanical ventilation in patients with acute respiratory distress syndrome. Intensive Care Med 2013; 39: 2056-2057.

8 Wiesner O, Hadem J, Sommer W, et al. Extracorporeal membrane oxygenation in a nonintubated patient with acute respiratory distress syndrome. Eur Respir J 2012; 40: 1296-1298.

9 Azoulay E, Roux A, Vincent F, et al. A multivariable prediction model for Pneumocystis jirovecii pneumonia in hematology patients with acute respiratory failure. Am J Respir Crit Care Med 2018; 198: 1519-1526. 